\title{
Numerical simulation of runoff from extreme rainfall events in a mountain water catchment
}

\author{
J. Burguete ${ }^{1}$, P. García-Navarro ${ }^{1}$, and R. Aliod ${ }^{2}$ \\ ${ }^{1}$ Fluid Mechanics, CPS, University of Zaragoza, Spain \\ ${ }^{2}$ Fluid Mechanics, EUP Huesca, University of Zaragoza, Spain
}

Received: 21 August 2001 - Accepted: 26 October 2001

\begin{abstract}
A numerical model for unsteady shallow water flow over initially dry areas is applied to a case study in a small drainage area at the Spanish Ebro River basin. Several flood mitigation measures (reforestation, construction of a small reservoir and channelization) are simulated in the model in order to compare different extreme rainfall-runoff scenarios.
\end{abstract}

\section{Introduction}

Many problems of flood routing, river management and civil protection consist of the evaluation of the maximum water levels and discharges that may be attained at particular locations during the development of an exceptional meteorological event. There is another category of events of catastrophic nature whose effects also fall into the civil protection area. In all cases it is the prevision of the scenario subsequent to the appearance and transport of a great volume of liquid onto a lower water stream. The situation can also include the case in which the stream is originally dry. There are works based on scaled physical models of natural valleys, but they represent efforts that were too expensive and not devoid of difficulties. Therefore, there is a necessity to develop adequate numerical models that are able to reproduce situations originated by the irregularities of a non-prismatic and sometimes dry bed. It is also necessary to trace their applicability considering the difficulty of developing a model capable of producing solutions of the complete equations despite the irregular character of the river bed.

Several methods exist, accepted as standard procedures, that are able to model the rainfall-runoff process. In general, they use data from precipitation records and soil physical characteristics to estimate the rainfall excess leading to surface runoff. Assuming that surface runoff eventually concentrates at the watershed outlet, provided that the effective

Correspondence to: P. García-Navarro

(pigar@posta.unizar.es) rainfall duration is longer than the basin time of concentration, those techniques supply a surface discharge flow hydrograph as the response of the system to the storm. The hydrograph, representing the variation of the discharge at a point with respect to time, can then be routed along a valley (river or creek), to estimate the possible flooding occurrence at downstream points.

Hydrograph or flood routing has usually been treated, assuming that only the main direction is relevant of the valley. For many practical applications, it is accepted that the unsteady flow of water in a one-dimensional approach is governed by the shallow water (or St. Venant) equations. These represent the conservation of mass and momentum along the direction of the main flow. In a simple approach, some models are based on steady-state assumptions (HEC-RAS), leading to the backwater profile calculation for every value of the discharge. Dynamic routing involves the unsteady flow assumption and the resolution of the full dynamic equations. In the field of computational hydraulics, where the modelling can be dominated by the effects not only of source terms, but also of quantities, which vary spatially yet independently of the flow variables, it has traditionally been difficult to have only one method that is able to reproduce automatically any general situation. The numerical modelling of unsteady flow in rivers is a complicated task and the difficulties grow as the pretensions increase to obtain better quality or more general solutions.

Good methods exist, developed to deal with Gas Dynamics problems (Euler equations), that are able to cope with complex systems of discontinuities and shock waves (Toro, 2001; Roe, 1981). Among them, flux difference splitting methods are widely used for the numerical approximation of homogeneous conservation laws where the flux depends only on the conservative variables. Many upwind schemes have been reported successfully for flow in channels. However, their application to river flow is not so common in the literature and their adaptation to river hydraulics is hindered by the irregular topography (García-Navarro et al., 1999). The presence of extreme slopes, high roughness and strong changes in the 
irregular geometry represent a difficulty that can lead to important numerical errors presumably arising from the source terms of the equations. The extension of the basic techniques to cope with these two situations maintaining their original properties is not straightforward but has been addressed in previous works (García-Navarro et al., 1999; Burguete and García-Navarro, 2001) and is applied here.

In this paper, the main focus is on the application of a numerical technique to the simulation of the stream flow generated by the dynamical routing of a discharge hydrograph on a dry valley. The intention of this paper is to highlight the suitability of the numerical technique as an efficient hydraulic flow forecasting tool, even in difficult cases of transcritical flow over dry bed.

The hydrologic problem will first be outlined. Then, the equations governing overland flow motion will be described and some ideas concerning the numerical method applied will be presented. The results obtained in the form of previsions under different flood hypothesis, helping to support the decision of risk managers, will finally be shown.

\section{The hydrologic problem}

The examples selected to show the applicability of the numerical technique used in this work belong to a wider hydrologic/hydraulic numerical study performed on a series of creeks draining a rural area which was being transformed into an irrigated agriculture area. The study was carried out to analyze the feasibility of several remediation strategies intended to reduce the damage that overflow in the creeks due to extreme rainfall events could cause on the near fields. The necessary investment for the works and launching of pressure distribution and irrigation systems required an estimation of the risk due to flood events associated with rains likely to occur in the amortization period.

For that purpose, historical hydrologic data were collected and the rainfall-runoff events corresponding to a return period of 10 years were characterized. Two methods were used to define the subsequent discharge at the watershed outlet points: The Rational Method, producing maximum discharge values, and the Unit Hydrograph Method, allowing the construction of discharge flow evolution in time, i.e., discharge hydrographs (Chow et a., 1994). Looking for risk analysis in the worst scenario, the Unit Hydrograph Method was used in the present study for two main reasons: since significantly higher discharge peak values were predicted and because the lagging time between additive peak values was considered a key factor in the study.

The main characteristics of the draining system analyzed in this paper are displayed on the sketch shown in Fig. 1. Valdecarro can be identified as the main creek (about $12 \mathrm{~km}$ long), running from right to left in the figure, draining the upstream part and carrying rain waters to the downstream Arba de Luesia River. Five secondary and relatively short tributary creeks can also be identified.

\section{The mathematical model}

The dynamic open channel flow equations (shallow water equations) of practical application in Hydraulics can be written as the following 1D hyperbolic systems with source terms (Chow et al., 1994; Toro, 2001):

$\frac{\partial \boldsymbol{u}(x, t)}{\partial t}+\frac{d \boldsymbol{F}(x, \boldsymbol{u})}{d x}=\boldsymbol{H}(x, \boldsymbol{u})$

where

$$
\begin{gathered}
\boldsymbol{u}=\left(\begin{array}{l}
A \\
Q
\end{array}\right), \quad \boldsymbol{F}=\left(\begin{array}{c}
Q \\
\frac{Q^{2}}{A}+g I_{1}
\end{array}\right), \\
\boldsymbol{H}=\left(\begin{array}{c}
q_{L} \\
g\left[I_{2}+A\left(S_{0}-S_{f}\right)\right]
\end{array}\right)
\end{gathered}
$$

and where $Q$ is the discharge, $A$ is the wetted cross section, $q_{L}$ is the lateral discharge, $g$ is the acceleration of gravity and $S_{0}$ is the bed slope. $I_{1}$ and $I_{2}$ account for hydrostatic pressure forces

$$
\begin{aligned}
& I_{1}(x, A)=\int_{0}^{h(x, A)}[h(x, A)-z] \sigma(x, z) d z, \\
& I_{2}(x, A)=\int_{0}^{h(x, A)}[h(x, A)-z] \frac{\partial \sigma(x, z)}{\partial x} d z
\end{aligned}
$$

( $h$ being the water depth and $\sigma$ being the channel width at a position $z$ from the bottom) $S_{f}$ is associated with bed friction and represented by the empirical Manning law (Chanson, 1999):

$S_{f}=\frac{n^{2} Q^{2} P^{\frac{4}{3}}}{A^{\frac{10}{3}}}$,

where $n$ is the Manning coefficient of bed roughness, here assumed in SI units, and $P$ is the wetted perimeter.

From the equations in conservative form (1), it is possible to pass to an associated non-conservative form using

$$
\begin{aligned}
& \frac{d \boldsymbol{F}(x, \boldsymbol{u})}{d x}=\frac{\partial \boldsymbol{F}(x, \boldsymbol{u})}{\partial x}+\frac{\partial \boldsymbol{F}(x, \boldsymbol{u})}{\partial \boldsymbol{u}} \frac{\partial \boldsymbol{u}}{\partial x} \\
& =\frac{\partial \boldsymbol{F}(x, \boldsymbol{u})}{\partial x}+\mathbf{J}(x, \boldsymbol{u}) \frac{\partial \boldsymbol{u}}{\partial x},
\end{aligned}
$$

where $\mathbf{J}=\frac{\partial \boldsymbol{F}}{\partial \boldsymbol{u}}$ is the Jacobian matrix of the original system. Redefining the source term as

$\boldsymbol{H}^{\prime}(x, \boldsymbol{u})=\boldsymbol{H}(x, \boldsymbol{u})-\frac{\partial \boldsymbol{F}(x, \boldsymbol{u})}{\partial x}$

the non-conservative form is obtained:

$\frac{\partial \boldsymbol{u}(x, t)}{\partial t}+\mathbf{J}(x, \boldsymbol{u}) \frac{\partial \boldsymbol{u}(x, t)}{\partial x}=\boldsymbol{H}^{\prime}(x, \boldsymbol{u})$.

In the shallow water system of equations the following holds

$$
\begin{aligned}
& \frac{d h}{d x}=\frac{\partial h}{\partial x}+\frac{1}{B} \frac{\partial A}{\partial x} \\
& \frac{d I_{1}}{d x}=\frac{\partial I_{1}}{\partial x}+\frac{\partial I_{1}}{\partial A} \frac{\partial A}{\partial x}=I_{2}+A \frac{\partial h}{\partial x}+\frac{A}{B} \frac{\partial A}{\partial x}=I_{2}+A \frac{d h}{d x}
\end{aligned}
$$


and the Jacobian and new source terms of the nonconservative formulation (2) are

$\mathbf{J}=\left(\begin{array}{cc}0 & 1 \\ c^{2}-v^{2} & 2 v\end{array}\right)$

$\boldsymbol{H}^{\prime}=\left(\begin{array}{c}0 \\ \left.g A\left[S_{0}-S_{f}-\frac{d h}{d x}+\frac{1}{B} \frac{d A}{d x}\right]\right)\end{array}\right.$

with $B$ the width at the free surface, $c=\sqrt{g \frac{A}{B}}$ the celerity of infinitesimal surface waves and $v=\frac{Q}{A}$ the mean fluid velocity.

It is convenient to develop the characteristic form of the equations, important for the correct formulation of upwind schemes and boundary conditions. This form is obtained from a diagonalization of the Jacobian in (2). Calling $\mathbf{P}$ and $\mathbf{P}^{-1}$ the matrices that make diagonal $\mathbf{J}$,

$\mathbf{J}=\mathbf{P} \Lambda \mathbf{P}^{-1}, \quad \Lambda=\mathbf{P}^{-1} \mathbf{J P}$.

The diagonal matrix $\Lambda$ is formed by the eigenvalues of $\mathbf{J}$, and $\mathbf{P}$ is constructed with its eigenvectors. Let $\boldsymbol{w}$ be the set of variables (characteristic variables) that verify

$d \boldsymbol{u}=\mathbf{P} d \boldsymbol{w}, \quad d \boldsymbol{w}=\mathbf{P}^{-1} d \boldsymbol{u}$.

Then,

$\frac{\partial \boldsymbol{w}(x, t)}{\partial t}+\Lambda(x, \boldsymbol{w}) \frac{\partial \boldsymbol{w}(x, t)}{\partial x}=\mathbf{P}^{-1}(x, \boldsymbol{w}) \boldsymbol{H}^{\prime}(x, \boldsymbol{w})$.

For the shallow water equations, the above matrices are

$\mathbf{P}=\left(\begin{array}{cc}1 & 1 \\ v+c & v-c\end{array}\right), \quad \mathbf{P}^{-1}=\frac{1}{2 c}\left(\begin{array}{cc}c-v & 1 \\ c+v & -1\end{array}\right)$,

$\Lambda=\left(\begin{array}{cc}v+c & 0 \\ 0 & v-c\end{array}\right)$.

\section{Numerical method}

The conservation law (1) contains an important physical meaning. An spatial integration

$$
\begin{gathered}
\int_{0}^{L}\left(\frac{\partial \boldsymbol{u}}{\partial t}+\frac{d \boldsymbol{F}}{d x}\right) d x=\int_{0}^{L} \boldsymbol{H} d x \Rightarrow \int_{0}^{L} \frac{\partial \boldsymbol{u}}{\partial t} d x \\
=\boldsymbol{F}_{0}-\boldsymbol{F}_{L}+\int_{0}^{L} \boldsymbol{H} d x
\end{gathered}
$$

expresses that the time variation of the conserved variable in a given volume is equal to the difference between the incoming and the outgoing fluxes plus the contribution of the source term. When discretizing a conservation law of this kind, incorrect numerical approximations can lead to bad behaviour in the solution and unacceptable errors, which makes them useless techniques. Schemes properly approximating the conservation equation (5) are called conservative schemes (Toro (2001)).

A numerical flux $\boldsymbol{F}_{i}^{T}$ and a numerical source term $\boldsymbol{H}_{i}^{T}$ can be defined at the grid nodes. The difference in the flux between two nodes can be decomposed into parts affecting the nodes on the left and right. Schemes built in such a manner follow

$\delta \boldsymbol{F}_{i+\frac{1}{2}}^{T}=\boldsymbol{F}_{i+1}^{T}-\boldsymbol{F}_{i}^{T}=\delta \boldsymbol{F}_{i+\frac{1}{2}}^{R}+\delta \boldsymbol{F}_{i+\frac{1}{2}}^{L}$

$\frac{\Delta \boldsymbol{u}_{i}^{n}}{\Delta t}=\boldsymbol{H}_{i}^{T}-\frac{1}{\delta x}\left(\delta \boldsymbol{F}_{i+\frac{1}{2}}^{R}+\delta \boldsymbol{F}_{i-\frac{1}{2}}^{L}\right)$

and will be conservative (Burguete and García-Navarro P., 2001) since they produce a good approximation of (5), cancelling the contributions of the flux at the grid interfaces, since the global variation of the conserved variable is due only to the source terms and to the flux at the boundaries. $\Delta$ will be used for time increments $\Delta f_{i}^{n}=f_{i}^{n+1}-f_{i}^{n}$, and $\delta$ for spatial increments $\delta f_{i+\frac{1}{2}}^{n}=f_{i+1}^{n}-f_{i}^{n}$.

$\sum_{i=1}^{N} \frac{\Delta \boldsymbol{u}_{i}^{n}}{\Delta t} \delta x=\frac{\Delta}{\Delta t} \sum_{i=1}^{N} \boldsymbol{u}_{i}^{n} \delta x \approx \frac{\partial}{\partial t} \int_{x_{1}}^{x_{N}} \boldsymbol{u} d x$

$\sum_{i=1}^{N}\left[\boldsymbol{H}_{i}^{T} \delta x-\left(\delta \boldsymbol{F}_{i+\frac{1}{2}}^{R}+\delta \boldsymbol{F}_{i-\frac{1}{2}}^{L}\right)\right]$
$=\sum_{i=1}^{N}\left[\boldsymbol{H}_{i}^{T} \delta x\right]+\boldsymbol{F}_{1}^{T}-\boldsymbol{F}_{N}^{T} \approx \boldsymbol{F}_{1}-\boldsymbol{F}_{N}+\int_{x_{1}}^{x_{N}} \boldsymbol{H} d x$

In addition, and following evidences from previous works (LeVeque, 1998; García-Navarro and Vázquez Cendón, 2000; Vázquez-Cendón, 1999), we consider a non-centered contribution of the source terms

$\boldsymbol{H}_{i+\frac{1}{2}}^{T}=\boldsymbol{H}_{i+\frac{1}{2}}^{R}+\boldsymbol{H}_{i+\frac{1}{2}}^{L}$

so that the following formulation for the conservative scheme is proposed

$\frac{\Delta \boldsymbol{u}_{i}^{n}}{\Delta t}=\left(\boldsymbol{H}-\frac{\delta \boldsymbol{F}}{\delta x}\right)_{i-\frac{1}{2}}^{L}+\left(\boldsymbol{H}-\frac{\delta \boldsymbol{F}}{\delta x}\right)_{i+\frac{1}{2}}^{R}$.

Defining $\boldsymbol{G}$ as

$\boldsymbol{G}_{i+1 / 2} \equiv\left(\boldsymbol{H}-\frac{\delta \boldsymbol{F}}{\delta x}\right)_{i+\frac{1}{2}} \equiv\left(\boldsymbol{H}^{\prime}-\mathbf{J} \frac{\delta \boldsymbol{u}}{\delta x}\right)_{i+\frac{1}{2}}$

$\frac{\Delta \boldsymbol{u}_{i}^{n}}{\Delta t}=\boldsymbol{G}_{i-\frac{1}{2}}^{L}+\boldsymbol{G}_{i+\frac{1}{2}}^{R}$

where the decomposition in the left and right parts has to be defined in every numerical scheme.

For the applications presented in this work, a second order in space and time upwind TVD scheme has been used. The choice is justified by a previous numerical study on the performance of a series of methods, which gave as a conclusion that this scheme was the one providing the best performance for unsteady flow over irregular valleys. In this scheme, the above decomposition is identified with left $\left(\boldsymbol{G}^{-}\right)$and right $\left(\boldsymbol{G}^{+}\right)$moving contributions: 


$$
\begin{aligned}
& \boldsymbol{G}_{i+\frac{1}{2}}=\left(\boldsymbol{G}^{-}\right)_{i+\frac{1}{2}}^{n}+\frac{\Delta t}{2}\left\{\left[\Psi^{+}\left(1-\frac{\Delta t}{\delta x} \mathbf{J}^{+}\right) \boldsymbol{G}^{+}\right]_{i-\frac{1}{2}}^{n}\right. \\
& \left.-\left[\Psi^{-}\left(1+\frac{\Delta t}{\delta x} \mathbf{J}^{-}\right) \boldsymbol{G}^{-}\right]_{i+\frac{3}{2}}^{n}\right\} \\
& \boldsymbol{G}_{i+\frac{1}{2}}^{L}=\left(\boldsymbol{G}^{-}\right)_{i+\frac{1}{2}}^{n}+\frac{\Delta t}{2}\left\{\left[\Psi^{-}\left(1+\frac{\Delta t}{\delta x} \mathbf{J}^{-}\right) \boldsymbol{G}^{-}\right]_{i+\frac{3}{2}}^{n}\right. \\
& \left.-\left[\Psi^{+}\left(1-\frac{\Delta t}{\delta x} \mathbf{J}^{+}\right) \boldsymbol{G}^{+}\right]_{i-\frac{1}{2}}^{n}\right\} \\
& \mathbf{J}^{ \pm}=\mathbf{P}\left(\begin{array}{cc}
\frac{1 \pm \operatorname{sign}(v+c)}{2} & 0 \\
0 & \frac{1 \pm \operatorname{sign}(v-c)}{2}
\end{array}\right) \mathbf{P}^{-1} \mathbf{J} \\
& \boldsymbol{G}^{ \pm}=\mathbf{P}\left(\begin{array}{cc}
\frac{1 \pm \operatorname{sign}(v+c)}{2} & 0 \\
0 & \frac{1 \pm \operatorname{sign}(v-c)}{2}
\end{array}\right) \mathbf{P}^{-1} \boldsymbol{G} .
\end{aligned}
$$

Making an implicit treatment of the source term, necessary to avoid the numerical instabilities produced by dominant source terms, the scheme is:

$$
\begin{aligned}
& \left(1-\mathbf{K}_{i}^{n} \frac{\Delta t}{2}\right) \Delta \boldsymbol{u}_{i}^{n}=\Delta t\left[\left(\boldsymbol{G}^{+}\right)_{i-\frac{1}{2}}^{n}+\left(\boldsymbol{G}^{-}\right)_{i+\frac{1}{2}}^{n}\right] \\
& +\frac{\Delta t}{2}\left\{\left[\Psi^{+}\left(1-\frac{\Delta t}{\delta x} \mathbf{J}^{+}\right) \boldsymbol{G}^{+}\right]_{i-\frac{1}{2}}^{n}\right. \\
& -\left[\Psi^{+}\left(1-\frac{\Delta t}{\delta x} \mathbf{J}^{+}\right) \boldsymbol{G}^{+}\right]_{i+\frac{3}{2}}^{n} \\
& +\left[\Psi^{-}\left(1+\frac{\Delta t}{\delta x} \mathbf{J}^{-}\right) \boldsymbol{G}^{-}\right]_{i+\frac{1}{2}}^{n} \\
& \left.-\left[\Psi^{-}\left(1+\frac{\Delta t}{\delta x} \mathbf{J}^{-}\right) \boldsymbol{G}^{-}\right]_{i+\frac{3}{2}}^{n}\right\}
\end{aligned}
$$

with $\mathbf{K}$ the Jacobian of the source term and $\Psi$ the limiting function matrix necessary to avoid numerical oscillations. For more details on this scheme, see Burguete and GarcíaNavarro P. (2001).

\subsection{Numerical boundary conditions}

The theory of characteristics supplies a rigorous rule for the numerical treatment of the boundary conditions at the upstream and downstream ends. In case of subcritical flow, at every boundary, one external or physical boundary condition is required and the numerical scheme must provide another one. On the other hand, if the flow is supercritical, two external boundary conditions need to be imposed at the inlet (upstream), whereas the numerical scheme will provide two numerical boundary conditions at the outlet (downstream).
In the case study considered in this work, the discharge hydrograph from the hydrologic analysis of the rainfall represents one physical boundary condition to be imposed upstream. For the physical properties at the downstream end of the creek, no representative physical boundary condition was known. The Arba de Luesia is a river of highly variable regime, hence no representative water level could be specified at the junction point. This, together with the fact that the torrent is usually dry, led us to the option of modelling the torrent downstream point as a free-flowing section. The method proposed here always supplies two conditions at the outlet and one condition at the upstream end so that both subcritical and supercritical flows can be dealt with appropriately.

The method is based on a very important physical principle: the increment of mass in the whole system in a time interval is the result of the entering mass flow minus the leaving mass flow during that period of time. When using a conservative numerical scheme, this physical principle provides a way to reach two objectives: first, to allow for a null mass balance error and second, to supply numerical boundary conditions.

In one time step, the numerical scheme as defined in (10) supplies updated values for all the nodal variables. We will denote them the by $u_{i}^{s}$, in particular, for the cross section, $A_{i}^{s}$, and the associated volume increment is

$$
\Delta M=\sum_{i=1}^{N}\left(A_{i}^{s}-A_{i}^{n}\right) \delta x .
$$

When (10) is applied to calculate the updated values $A_{i}^{s}$ and this is used in the volume increment calculation, the above expression is

$\Delta M=\left(Q_{1}^{n}-Q_{N}^{n}\right) \Delta t$

and the difference with respect to the net incoming flow rate is the volume error of the numerical scheme. It can be seen as if the scheme was generating a numerical inflow volume $V_{\text {in }}^{\text {num }}=Q_{1}^{n} \Delta t$ and a numerical outflow volume $V_{\text {out }}^{\text {num }}=$ $Q_{N}^{n} \Delta t$. In order to achieve perfect volume conservation, if the upstream physical boundary condition is $Q_{1}^{n+1}$ at the inlet, and it is assumed that the physical volume entering during one time step is $V_{i n}^{p h y}=\frac{1}{2}\left(Q_{1}^{n+1}+Q_{1}^{n}\right) \Delta t$, then the corrected value for the wetted section upstream $A_{1}^{n+1}$ is

$A_{1}^{n+1}=A_{1}^{s}+\frac{V_{i n}^{p h y}-V_{i n}^{n u m}}{\delta x}=A_{1}^{s}+\frac{1}{2}\left(Q_{1}^{n+1}-Q_{1}^{n}\right) \frac{\Delta t}{\delta x}$

and for the wetted section downstream $A_{N}^{n+1}$ is

$A_{N}^{n+1}=A_{N}^{s}-\frac{1}{2}\left(Q_{N}^{n+1}-Q_{N}^{n}\right) \frac{\Delta t}{\delta x}$

in the case of having supercritical flow, upstream:

$A_{1}^{n+1}=\frac{V_{\text {in }}^{\text {phy }}-V_{\text {in }}^{\text {num }}}{\delta x}=\frac{1}{2}\left(Q_{1}^{n+1}-Q_{1}^{n}\right) \frac{\Delta t}{\delta x}$ 


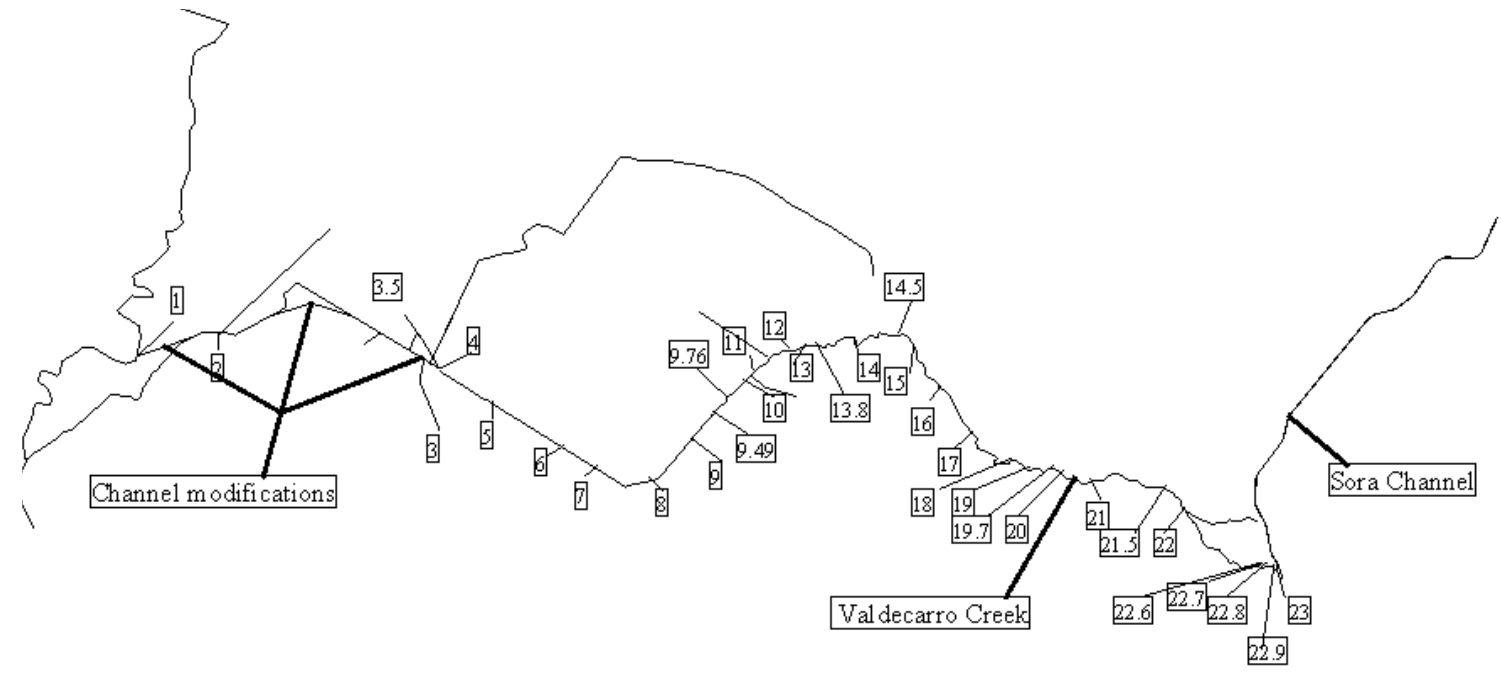

Fig. 1. Plan view of the basin.

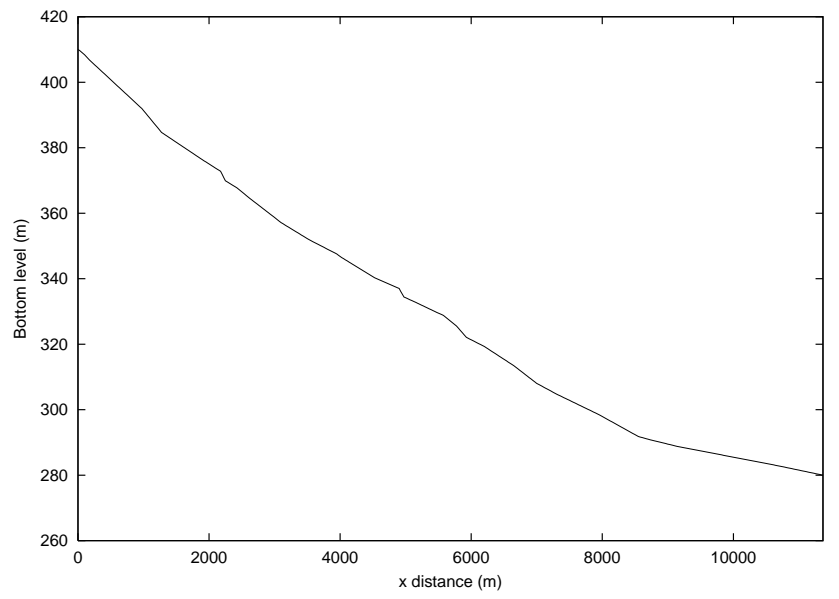

Fig. 2. Bottom level variation of the creek along the main direction.

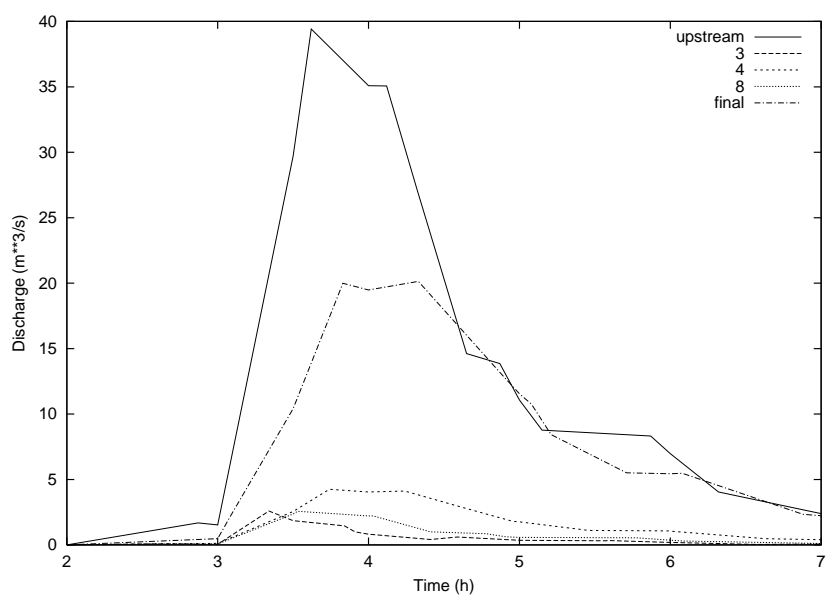

Fig. 3. Discharge hydrographs at sections for the basic case. and downstream

$A_{N}^{n+1}=A_{N}^{s}$

$Q_{N}^{n+1}=Q_{N}^{s}$

\subsection{Front advance over dry bed}

Unsteady shallow water flow over dry beds is at present one of the topics of research in computational hydraulics. One way to deal with this kind of flow is to use a moving computational mesh so that computation is only performed in the wet cells and the grid moves as the water front does. Suitable boundary conditions must be applied for the correct front tracking. A different approach consists of a thorough calculation of the front position as it advances over a computational mesh covering all the physical domain and in which there are both wet and dry cells. Among the authors using this second methodology, and for numerical reasons, a very small value for water depth in the dry cells is frequently used in order to avoid zero depth values. The amount of this threshold is reported to be something between $10^{-3} \mathrm{~m}$ and $10^{-6} \mathrm{~m}$, not precised or justified in general.

On the other hand, the shallow water equations can be derived from the Navier-Stokes equations through a depth averaging (in 2D) or a cross section averaging (in 1D) process. The product of such averaging is a set of equations written in terms of wetted cross section size and average velocity. The information concerning the original velocity profile is therefore reduced to a friction source term which takes into account the wall tangential stress and other viscous effects. This is usually modelled by means of a Manning type formula which actually derives from the viscous nature of the velocity profile.

A way to give physical meaning to the threshold value has been attempted in our work. In nature, rivers and streams do not have a regular bottom roughness, but the bed consists 


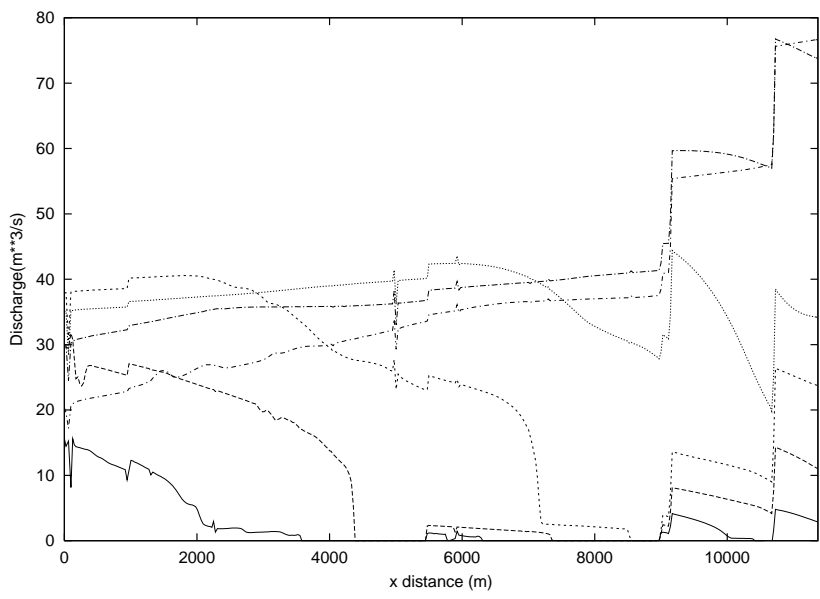

Fig. 4. Discharge profiles at times $t=3: 00 \mathrm{~h}, 3: 15 \mathrm{~h}, 3: 30 \mathrm{~h}, 3: 45 \mathrm{~h}$, $4: 00 \mathrm{~h}$ and $4: 15 \mathrm{~h}$ for the basic case.

of unsorted sand, gravel and rocks. Numerous researchers in the past have tried to relate the equivalent roughness height to a characteristic grain size, such as the median grain size $d_{50}$, leading to a general rule of proportionality between them. On the other hand, it is well known that in torrents and mountain streams the size of the boulders can be of the order of magnitude of the flow depth. In those cases, the flow resistance is mainly due to form drag and energy dissipation more than skin friction. Neither Chezy nor Manning formulae are adequate to estimate the friction losses, and experimental investigations should be performed beforehand. Due to the impossibility of carrying out such field experiments in most cases, a compromise is required.

The option chosen in this work is based on the empirical correlation proposed by Strickler in 1923 (Chanson, 1999) for the Manning coefficient in rivers

$n=0.041 d_{50}^{1 / 6}$

Given an estimation for the global or local Manning coefficient, the above relation supplies the order of magnitude of $d_{50}$. In our model, this value is used as the minimum water depth required at the front position to allow front advance. For water depths below that value, water is forced to stop and accumulate.

\section{Risk remediation strategies and numerical results}

The numerical method outlined in Sect. 4 was used to solve the equations presented in Sect. 3 and therefore to simulate the flood wave generated by the rainfall-runoff hydrographs along the Valdecarro Creek during a 5 hour flooding event. For this simulation the discharge hydrograph at the upstream end of the creek was used as physical boundary condition and subsequent hydrographs, entering Valdecarro Creek through its confluences, were considered as lateral discharges. A general sketch of the region is presented in Fig. 1, where Valdecarro Creek is represented entering by the right side of the

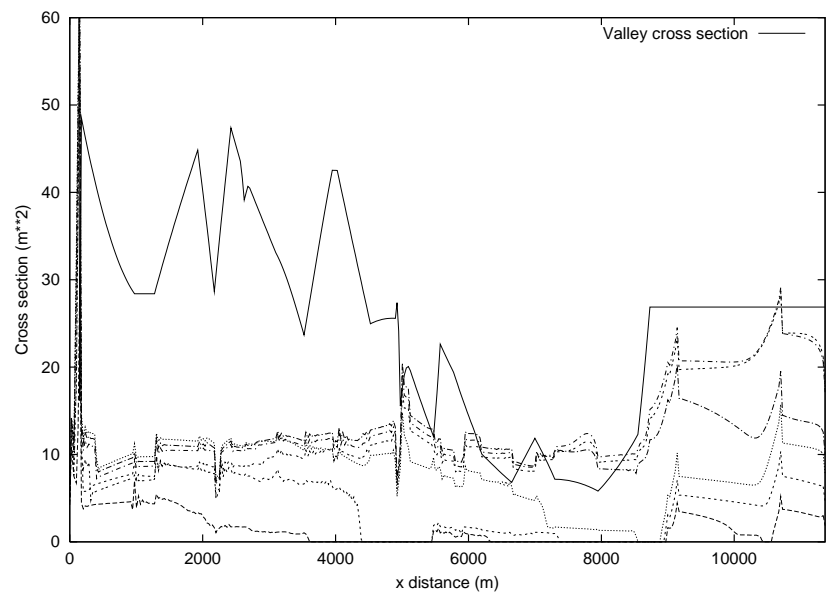

Fig. 5. Valley cross section (continuous line) and water cross section profiles at times $t=3: 00 \mathrm{~h}, 3: 15 \mathrm{~h}, 3: 30 \mathrm{~h}, 3: 45 \mathrm{~h} 4: 00 \mathrm{~h}$ and $4: 15 \mathrm{~h}$ for the basic case.

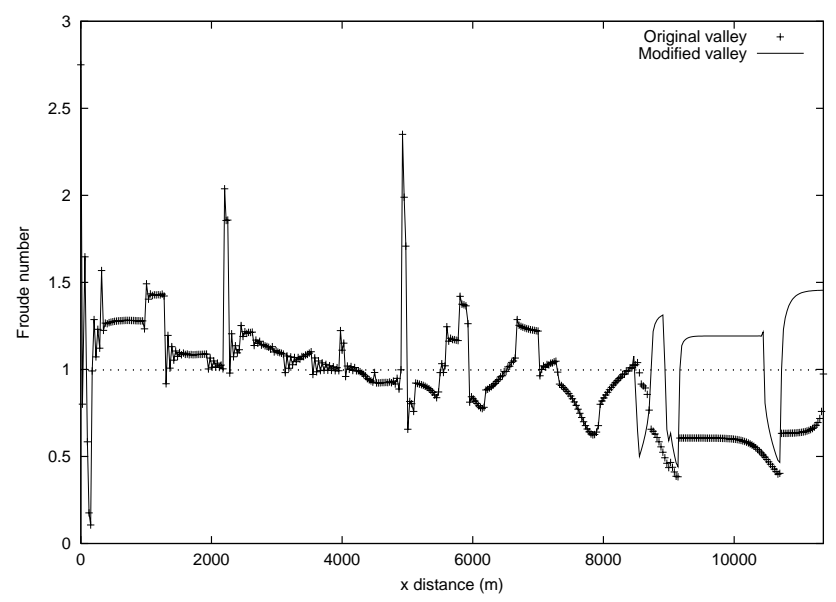

Fig. 6. Froude number distribution at time $t=4: 00 \mathrm{~h}$ in the basic case.

figure, running over about $12 \mathrm{~km}$ and joining Arba de Luesia River at the left part of the figure. One of the main features of the creek topography is the high variability of the cross section shape along the main axis. The valley is wide, devoid of bank vegetation and well divided into a curved main channel and a flood plain in the upstream part (from 0 to $5 \mathrm{~km}$ approximately). In particular, there is a very wide reach in the first 200 meters of creek. At $x=5 \mathrm{Km}$ approximately, a transition from composed to single cross section shape of minor capacity can be noticed. From $5 \mathrm{~km}$ to $8 \mathrm{~km}$ this tendency continues and, at the same time, considerably more vegetation covering the banks can be found. The lower part of the creek, from $8 \mathrm{~km}$ ahead, can be considered an artificially shaped triangular channel of decreasing, tending to zero, capacity. The torrent ends near the river in a plain crossed by a road. From the beginning of this study, the lower part was identified as problematic and a preliminary decision of performing a channelization in the last $3 \mathrm{~km}$ was made. Figure 2 shows the bed level variations. As for the roughness char- 


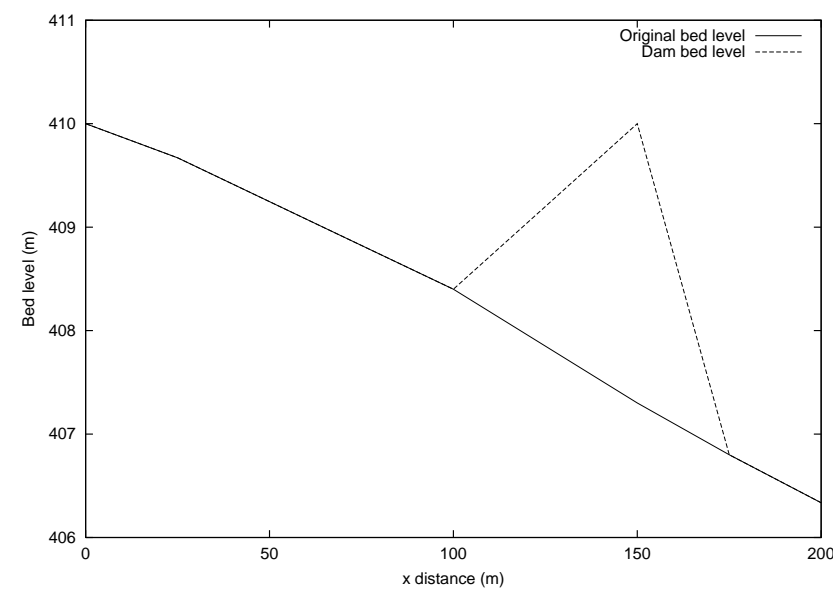

Fig. 7. Detail of the dam for the internal reservoir modelling (s1).

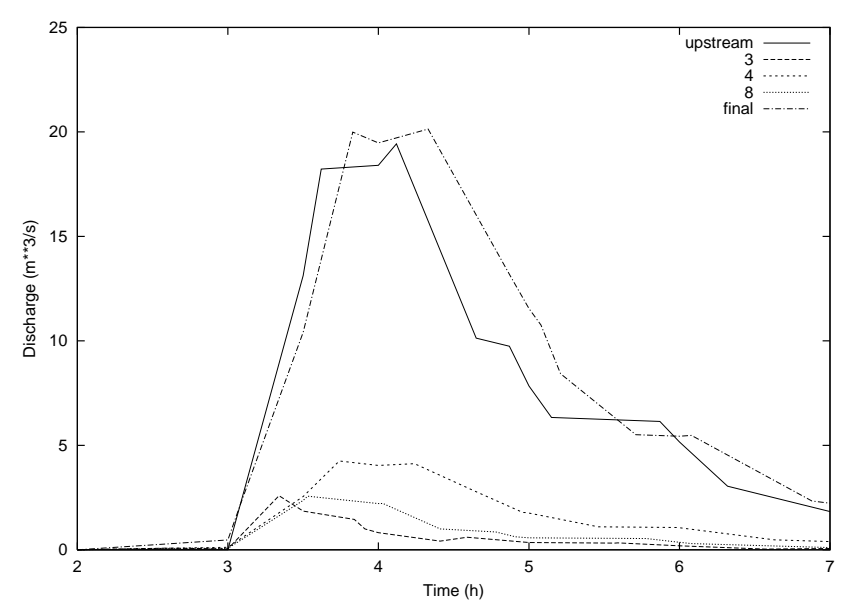

Fig. 8. Discharge hydrographs at different sections for the upstream reforestation case (s2).

acterization, all the reaches were given an average value of Manning's $n=0.03$.

In a first calculation, the basic magnitudes defining the torrent were used to evaluate the extent of the flooding in the presence of the actual physical and hydrologic conditions, as well as the mentioned channelization. The valley cross section shape was known up to a limit in width. From that point, vertical walls were assumed, i.e., no real flood plain was simulated. The results indicated that the banks were not able to convey the water in the middle part under the assumed rain hypothesis. The discharge hydrographs deduced from the hydrologic hypothesis are shown in Fig. 3 where the flow discharge law versus time has been plotted for a time interval of $5 \mathrm{~h}$, the period considered in this study. The discharge entering the creek by the upstream section has been plotted, together with the hydrographs joining the stream along the creek. This results are shown in Fig. 5, where the valley cross section is plotted along the $x$ distance, together with several wetted cross sections corresponding to the time evolution of the flood event which

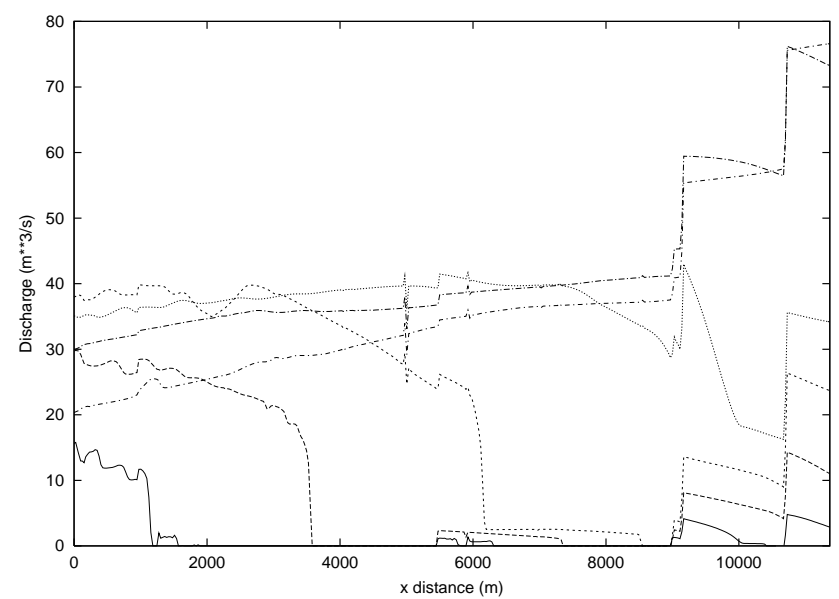

Fig. 9. Discharge profiles at times $t=3: 00 \mathrm{~h}, 3: 15 \mathrm{~h}, 3: 30 \mathrm{~h}, 3: 45 \mathrm{~h}$, $4: 00 \mathrm{~h}$ and $4: 15 \mathrm{~h}$ for the internal reservoir case (s1).

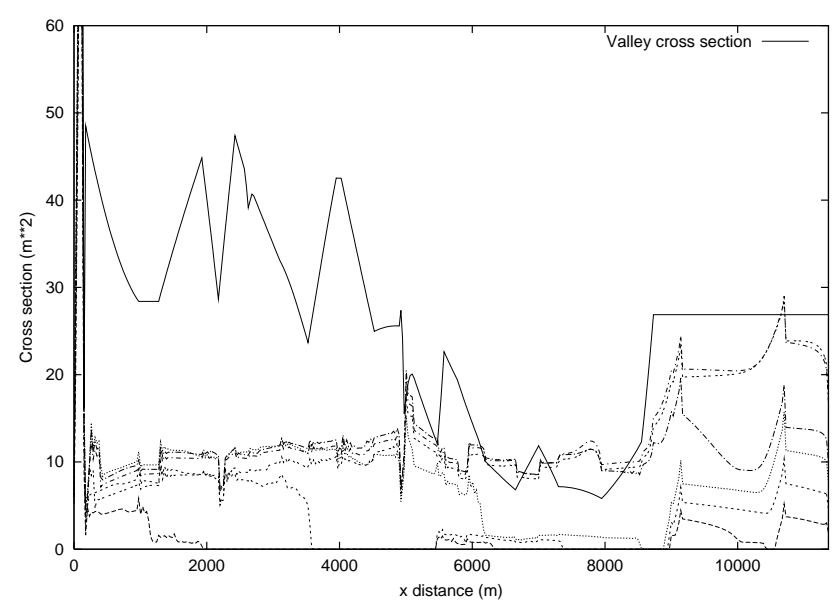

Fig. 10. Valley cross section (continuous line) and water cross section profiles at times $t=3: 00 \mathrm{~h}, 3: 15 \mathrm{~h}, 3: 30 \mathrm{~h}, 3: 45 \mathrm{~h}, 4: 00 \mathrm{~h}$ and $4: 15 \mathrm{~h}$ for the internal reservoir case (s1).

is supposed to last approximately $5 \mathrm{~h}$. Figure 4 displays discharge profiles at different times in order to follow not only the progression of the discharge flooding wave, but also the effect of the secondary hydrographs entering at later times through the tributary creeks. It is also worth noting the torrential character of the flow in this simulation, hence the inherent numerical difficulties, as indicated by the Froude number distribution along the valley at an intermediate time during the simulation in Fig. 6. This figure also indicates the supercritical regime at the downstream end.

The second step, and main bulk of this work, was the definition of different scenarios oriented to flood risk mitigation. In order to exploit the possibilities that CFD offers, several hypotheses were modelled to check their suitability and ability to reduce flood damage. The first was to model the effects of the construction of an in-line reservoir as storage structure at the upstream inlet by making use of the extremely wide natural cross section in that part. We shall call this solution 1 


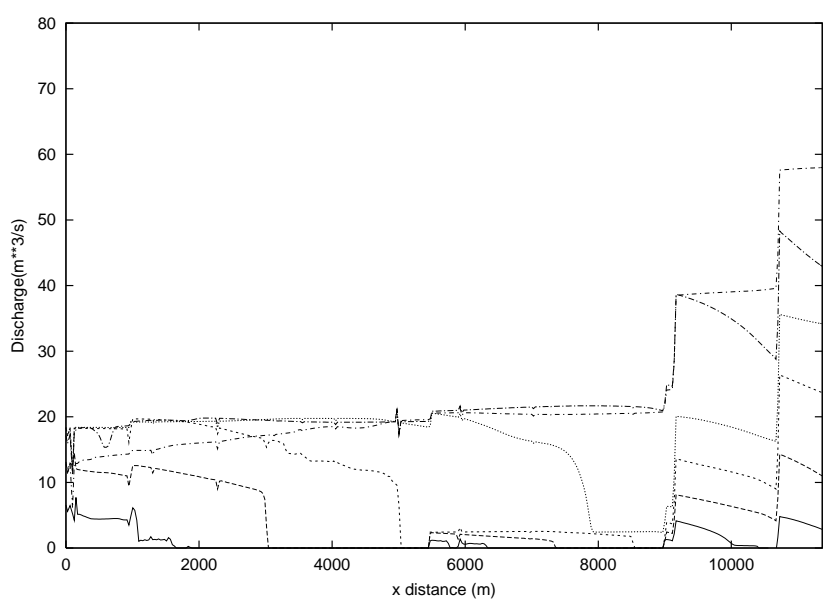

Fig. 11. Discharge profiles at times $t=3: 00 \mathrm{~h}, 3: 15 \mathrm{~h}, 3: 30 \mathrm{~h}, 3: 45 \mathrm{~h}$, 4:00 $\mathrm{h}$ and $4: 15 \mathrm{~h}$ for the reforestation case(s2).

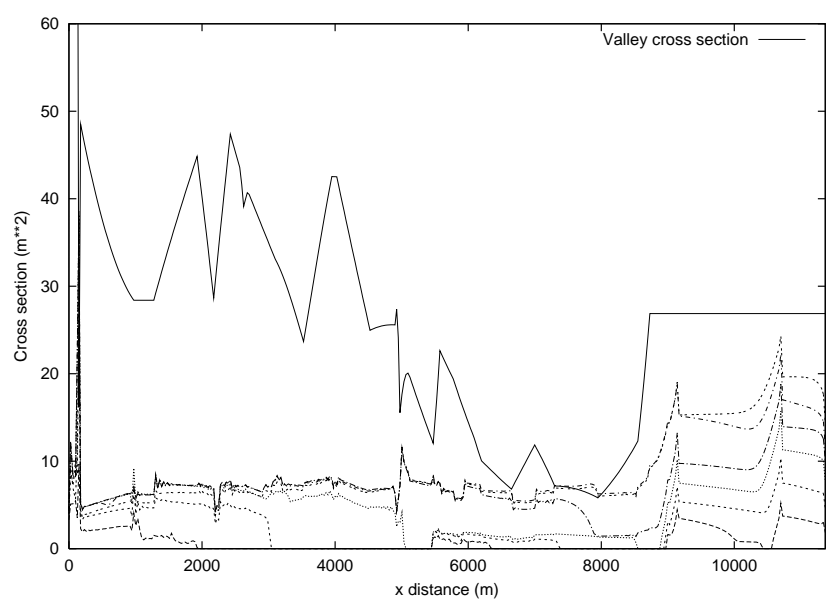

Fig. 12. Valley cross section (continuous line) and water cross section profiles at times $t=3: 00 \mathrm{~h}, 3: 15 \mathrm{~h}, 3: 30 \mathrm{~h}, 3: 45 \mathrm{~h}, 4: 00 \mathrm{~h}$ and $4: 15 \mathrm{~h}$ for the reforestation case(s2)

(s1). This reservoir was simulated in our model by means of a sufficiently rising bed level in the cross section at the location of the hypothetical dam. This detail is shown in Fig. 7, where the original bed level and the one modified to represent the dam location are plotted together. The storage capacity of this reservoir was estimated at around $10000 \mathrm{~m}^{3}$, requiring a filling time of $5 \mathrm{~min}$ at a rate of $35 \mathrm{~m}^{3} / \mathrm{s}$, the discharge value at the inlet at the peak of the storm. Figures 9 and 10 display the numerical results corresponding to this hypothesis for the wetted cross section and the discharge evolution, respectively. They show clearly that the modelled reservoir has no significant effect. The reservoir capacity is not enough to produce a noticeable damping of the peak discharge hydrograph.

In a different approach, the second flood mitigation measure introduced in our model was the hypothesis of upstream reforestation. This will be called solution 2 (s2). The assumption of a change in the land use and vegetation over the

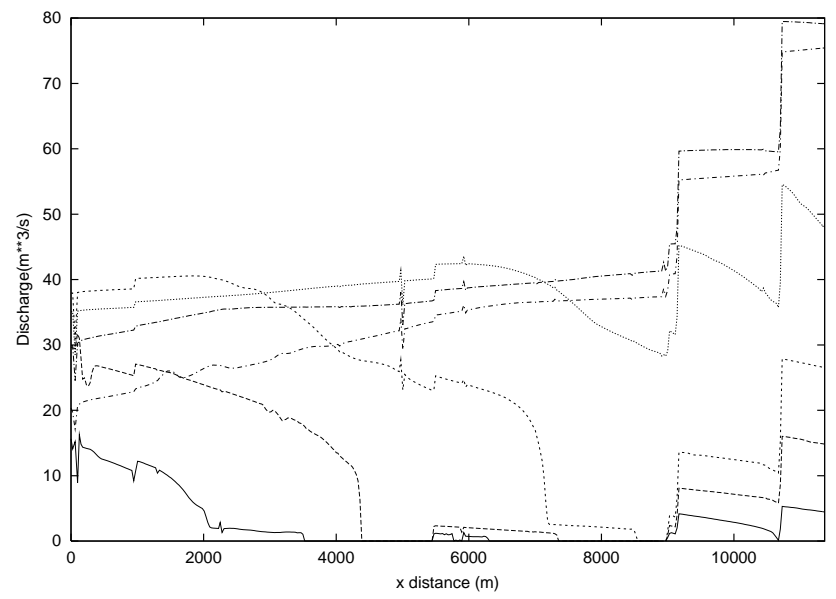

Fig. 13. Discharge profiles at times $t=3: 00 \mathrm{~h}, 3: 15 \mathrm{~h}, 3: 30 \mathrm{~h}, 3: 45 \mathrm{~h}$, 4:00 $\mathrm{h}$ and $4: 15 \mathrm{~h}$ for the bank clearing case(s3).

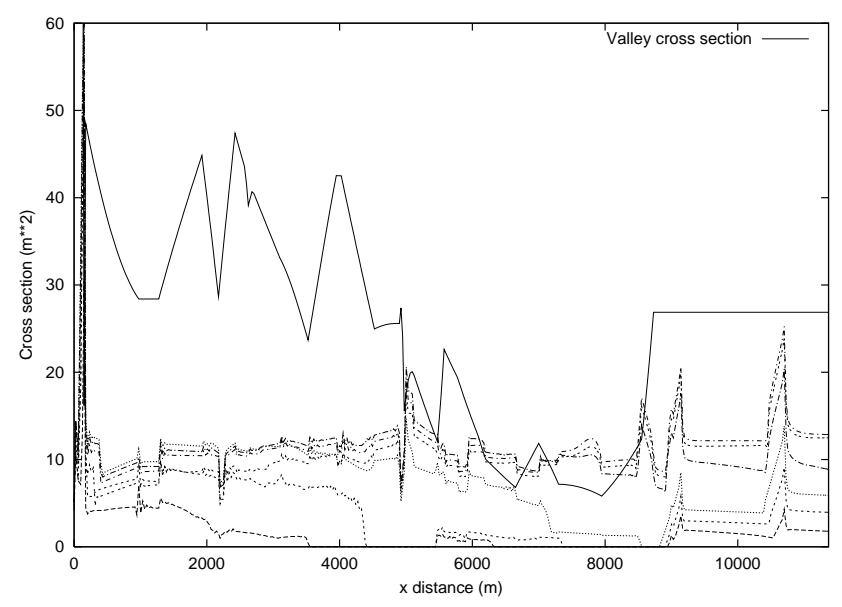

Fig. 14. Valley cross section (continuous line) and water cross section profiles at times $t=3: 00 \mathrm{~h}, 3: 15 \mathrm{~h}, 3: 30 \mathrm{~h}, 3: 45 \mathrm{~h}, 4: 00 \mathrm{~h}$ and $4: 15 \mathrm{~h}$ for the bank clearing case (s3).

area forming the basin, draining into the upper part of the creek, modified the parameters defining the soil infiltration capacity so that for identical rain conditions, smaller peak hydrographs resulted. This can be seen on the discharge hydrographs plotted in Fig. 8, where the upstream discharge hydrograph must be compared with the one in Fig. 3. The subsequent flood profiles, as obtained from the numerical routing, are displayed in Fig. 11. The main consequence was that under this hypothesis, the water depths were also reduced so that the preliminary channelization of the lower part can be considered sufficient to convey the flow from the assumed rain intensity.

As the third and last risk mitigation strategy, a roughness reduction in the channelized part was proposed by means of bank vegetation clearing and concrete cover. This will be referred to as solution 3 (s3). This option was simulated by means of a modified Manning roughness coefficient in the lower $3 \mathrm{~km}$, reducing the original $\mathrm{n}=0.03$ to $\mathrm{n}=0.015$. The 


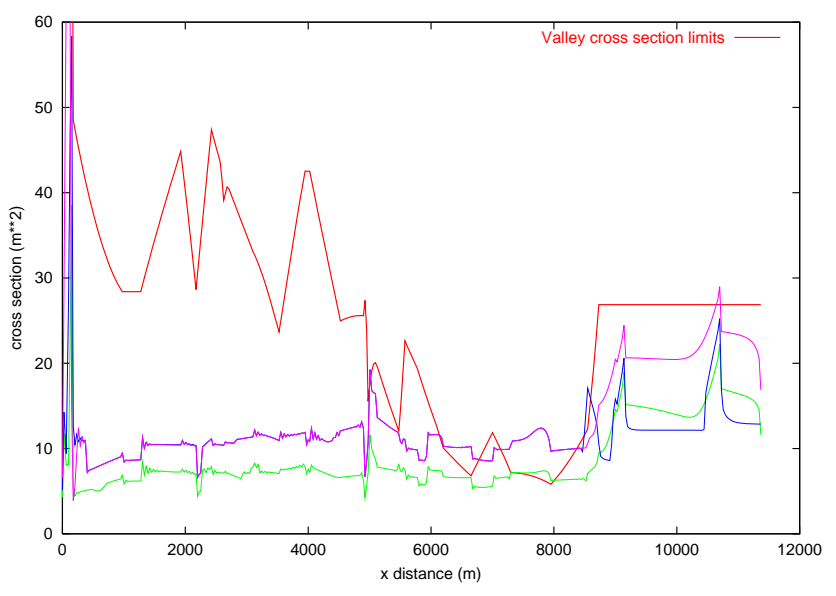

Fig. 15. Valley cross section (red line) and water cross section profiles at time $t=4: 00 \mathrm{~h}$ for $\mathrm{s} 1$ (pink), s2 (green) and s3 (blue).

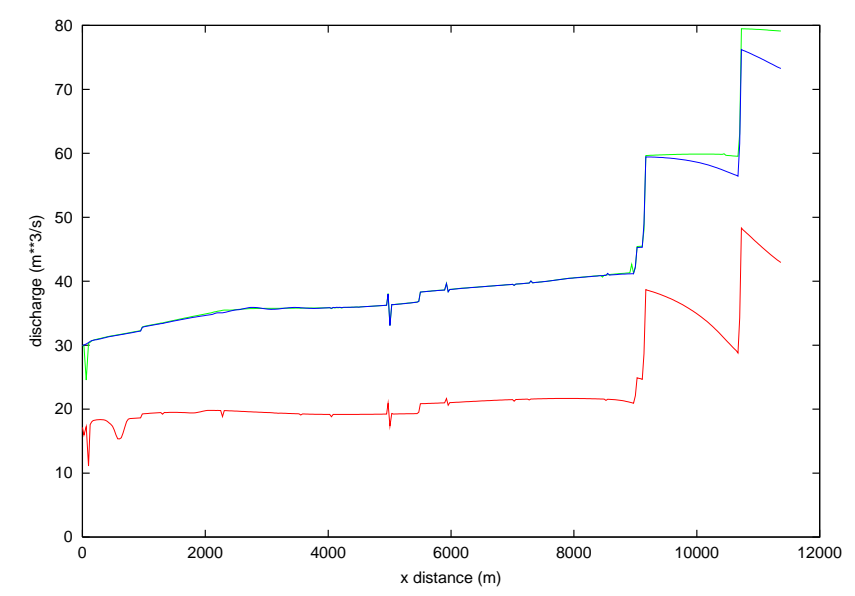

Fig. 16. Discharge distribution at time $t=4: 00 \mathrm{~h}$ for $\mathrm{s} 1$ (blue), s2 (red) and s3 (green).

results from this option led to a flow acceleration in that part with, consequently, smaller water depths and higher velocities and Froude numbers for the same discharge values as in the two first calculations. In some sense, the objective of preventing flooding on the surrounding areas is achieved with this option, but the supercritical flow involved at the downstream part of the stream renders the interest in the modification to be doubtful. The numerical results are plotted in Figs. 13 and 14. A direct comparison of the results obtained from the three hypotheses is shown in Fig. 15, where the maximum wetted cross section distributions are compared and in Fig. 16, where the maximum discharge distributions are compared.

\section{Conclusions}

A finite volume based numerical technique has been applied as a CFD tool for evaluating different flood risk remediation strategies in a low mountain area. The computational model solves the unsteady shallow water equations in the presence of dry bed and irregular topography. It has proved robust, useful and efficient as a predictive tool, being able to handle transcritical flow situations by keeping the mass error balance close to machine accuracy.

From the hydraulic/hydrologic point of view, the answers supplied by the computational model allow for the comparison in terms of usefulness and practical interest of different proposed alternatives. Among those modelled, it can be concluded that investments in internal reservoirs or valley channelization are not as effective as a modification in the land use and vegetation of the upstream area. This latest option seems to have the strongest influence in the reduction of flooding risks, since it directly affects the peak discharge hydrograph. On the other hand, bank clearing at the channelized lower part of the valley results in an increased conveyance capacity involving lower water depths but higher flow velocity and supercritical regime in some parts. This kind of flow, although having less risk of over flooding the banks, can damage the channel due to the inherent possibility to generate hydraulic jumps and turbulence.

Acknowledgements. This work could be performed thanks to the funding provided by the DGA. The collaboration of C. Estrada in the preliminary part of this study is greatly appreciated.

\section{References}

Burguete J. and García-Navarro P.: Efficient construction of highresolution TVD conservative schemes for equations with source terms. Application to shallow water flows, Int. J. for Numerical Methods in Fluids, 37, 209-248, 2001.

Chanson, H.: The hydraulics of open channel flow, Arnold, 1999.

Chow, V. T., Maidment D. R., and Mays L. W.: Applied Hydrology, McGraw Hill, 1994.

García-Navarro, P., Fras, A., and Villanueva, I.: Dam-break flow simulation: some results for one-dimensional models of real cases, J. of Hydrology, 216, 227-247, 1999.

García-Navarro, P., and Vázquez Cendón, M. E.: On numerical treatment of the source terms in the shallow water equations, Computers and Fluids, 126, 26-40, 2000.

Katopodes, N. and Schamber, D. R.: Applicability of Dam-break Flood Wave Models, J. of Hydraulic Engineering, 109, 5, 1983.

LeVeque, R. J.: Balancing source terms and flux gradients in high-resolution Godunov methods: the quasi-steady wavepropagation algorithm, J. of Computational Physics, 146, 1, 346365, 1998.

Roe, P. L.: Approximate Riemann solvers, parameter vectors, and difference schemes, Journal of Computational Physics, 43, 2, 357-372, 1981.

Toro, E.: Shock-Capturing Methods for Free-Surface Shallow Flows, Wiley, UK, 2001.

Vázquez-Cendón, M. E.: Improved treatment of source terms in upwind schemes for the shallow water equations in channels with irregular geometry, J. of Computational Physics, 148, 2, 497526, 1999. 